\title{
Remote Controller on Smartphone for Shorter Waiting Time in Elevator System
}

\author{
[ Hiroaki Higaki ]
}

\begin{abstract}
In a large-scale high-rise building, elevator systems with multiple elevator cars supports mobility of users. Since current elevator controller systems only achieve user's requirements as the existence of requests of mobility directions in each floor, it is difficult for multiple elevator cars to stop at the same floor simultaneously even if there exist unevenly distributed requests in some specific floors. This results in low service ratio causing numbers of bumped passengers and in longer waiting time duration of the users. This paper proposes a novel method for registrations of user's requests through a smartphone application. Due to achievement of mobility request of each user through his/her personal device, the elevator controller system acquires numbers and distribution of individual mobility requests and detailed control of elevator cars including simultaneous stops of multiple elevator cars at the same floor is realized. Simulation experiments show the proposed method provides higher service ratio and shorter waiting time of users than the conventional methods.
\end{abstract}

Keywords-Elevator Systems, Smartphones, Beacon Stations, Protocol.

\section{Introduction}

Elevator systems with multiple elevator cars become popular and are widely used as increase of requirements for largescale high-rise buildings. Mobility requirements of users are dynamically made and are changed moment by moment. In school buildings, office buildings, shopping malls and so on, in the specified time duration called an up-peak time, large number of users make mobility requests from a few specified floors. Especially, such crowded floors adjoiningly exists, full elevator cars might successively pass such crowded floors and user's waiting time for available elevator cars gets longer since the currently widely used up-down button device only notifies the existence of mobility requests of users in each floor specified only by mobility direction, i.e. up or down, to the elevator controller system.

\section{RELATED WORKS}

One of the most widely available user interfaces of elevator systems is combination of the call registration device with updown button installed in each floor and the destination floor registration device in the elevator cars as shown in Figure 1 [2], [3]. Waiting users in an elevator hall in each floor can only notify their requests to the elevator systems by the

Hiroaki Higaki

Tokyo Denki University

Japan mobility direction, i.e. up or down, by pushing the up-down button. Thus, the elevator controller system only gets the existence of mobility requests for up and down directions in each floor and does not get their destination floor information and the number of requests. Therefore, from a certain floor, multiple users whose destination floors are the same might get into different elevator cars and numbers of floors at which the elevator cars stop increases.

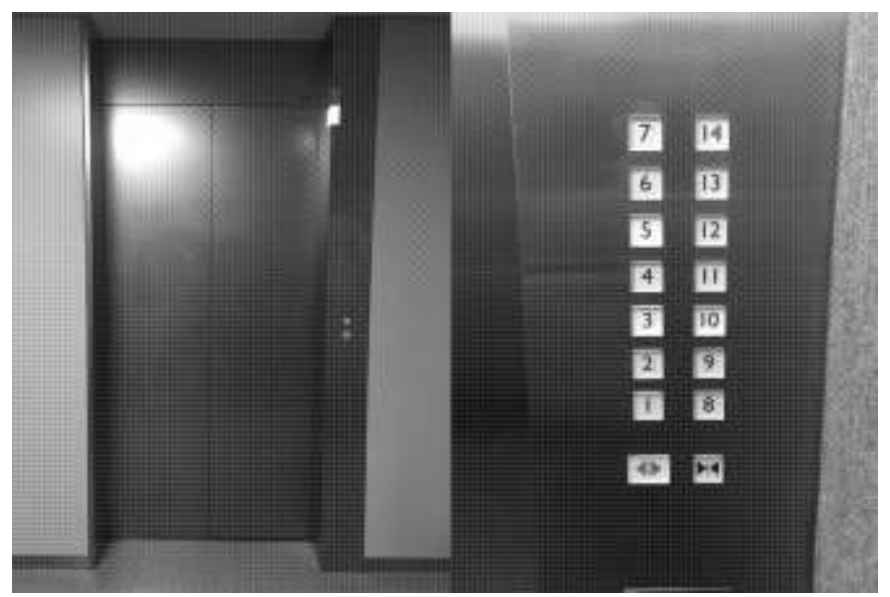

Figure 1. Up-Down Button Device

In result, longer mobility time duration is required. In order to solve this problem, advance destination registration devices have been proposed [1]. Here, the destination floor register device is installed in an elevator hall in each floor as shown in Figure 2. By using this device, the waiting users notify their destination floors instead of their mobility directions to the elevator controller systems. In addition, since the elevator controller system achieves the information of user's requests as a set of tuples of their current floor and destination one, it calculates and shows the specified elevator cars for the users to get into through the device. The users get into the specified elevator cars and the numbers of floors at which the elevator cars stop, which results in the reduction of mobility time duration of the users. For example, a user at the ground floor push the button of the 5th floor which is his/her destination floor to notify his/her request to the elevator controller system. Then, the system calculates the schedule of the elevator cars and shows the specified elevator car, e.g. elevator A in figure 2 , to the user to get into.

This method is expected to work well under the assumption that the elevator system is not so much crowded. However, in cases that an elevator hall is so much crowded, it does not work well. Since this system requires the waiting users to push their destination buttons, due to low accessibility to the advance destination registration devices in Figure 2, it is difficult for the users to register their destination floor to the 
elevator controller systems. In addition, the correspondance between their destination floors and the elevator cars to get into is also shown by using the device, its low visibility in the crowded elevator halls decreases its usability. Finally, since the elevator controller system cannot get the number of waiting users in each floor, the performance of the elevator controller systems cannot be improved enough and the low service ratio problem discussed later cannot be solved.

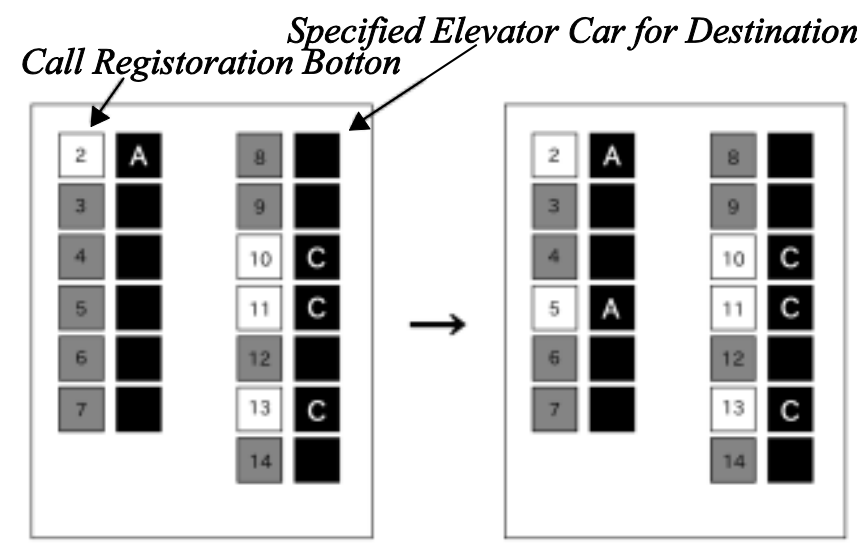

Figure 2. Advance Destination Registration Device.

In recent towns, underground passages and pedestrian decks have been widely constructed for pedestrians and vehicles to be separated for their safety. As a result, for the up-peak duration such as the peak time of commutation, consecutive multiple floors such as underground, ground and the first floors become crowded with the elevator users. In schools, at the beginning and the ending of lectures, elevator halls become full of students waiting for their elevator cars. In this case, since the current elevator controller systems cannot get the number of waiting users in each floor and the currently available capacity of each elevator cars for additional users, while an elevator car stops at a certain floor for users to get into and/or out of the elevator car, other elevator cars with the same mobility directions pass through the floor even if there might be bumped passengers in this floor and the elevator cars

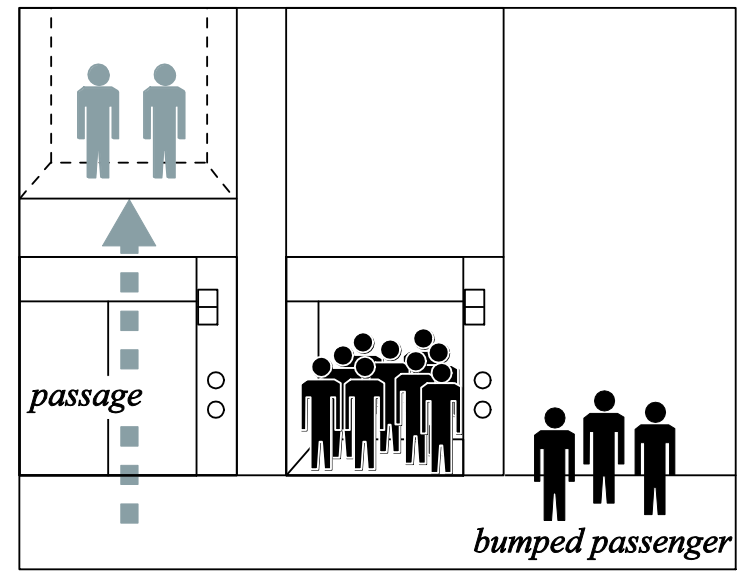

Figure 3. Low Service Ratio due to Unreasonable Passage. have enough available capacity for them as shown in Figure 3. This results in the low service ratio and the longer waiting time of users. In order to avoid such unreasonable passage of the elevator cars, number of waiting users in each floor and the destination of the waiting users are required to be achieved by the elevator controller systems. However, it is difficult for the currently used stationary registration devices for user's requests to satisfy the requirements.

\section{PROPOSAL}

This paper proposes a novel user interface between users waiting for elevator cars and the elevator controller systems implemented not on stationary devices but on smartphones of the users. Each user notifies their requests for elevator cars to the elevator controller system with his/her destination floor through his/her smartphone and his/her specified elevator car is shown on the screen of his/her smartphone. As indicated in [4], in the currently working and/or the future expected ICT systems, smartphones of the users are most reasonable userinterfaces to the systems since users are expected to always carry them. That is, a smartphone might becomes a universal interface to all the ICT systems supporting our everyday lives. Since each user notifies their requests with his/her destination floor to the elevator controller system, the system acquires the number of waiting users in each floor and the number of users for each destination floor. In addition, the specified elevator car is indicated to each user through his/her smartphone, the elevator controller system reasonably assigns the elevator cars to the waiting users to reduce the number of floors at which the elevator cars stop and the moving time duration of the users are also expected to be reduced. For example, as shown in Figure 4, different from the currently available elevator systems as shown in Figure 3, even if an elevator car has already stopped at a certain floor, it is possible for another elevator car to stop at the same floor for the waiting users to get into. Thus, the opportunities and the number of bumped passengers in this floor are expected to be reduced.

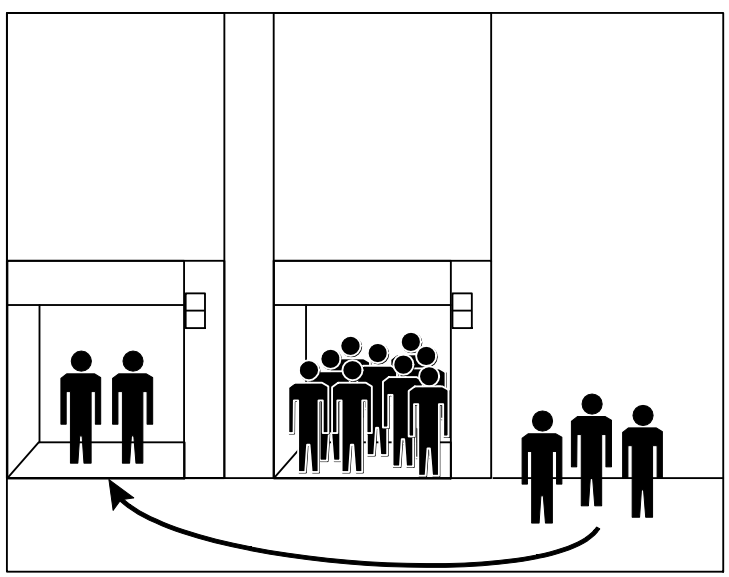

Figure 4. Improved Service Ratio due to Avoidance of Unreasonable Passage. 
Our proposed elevator system consists of an remote controller management server connected to the conventional elevator controller system through the Internet, stationary wireless beacon station in an elevator hall in each floor and the user's smartphones into which the remote controller application software has been installed as shown in Figure 5. A stationary wireless beacon station periodically transmits beacon messages to smartphones in the same elevator hall to notify the ID of the elevator hall. Since the transmission range of the beacon message is enough small such as $10 \mathrm{~m}$ that all and only the smartphones in the same floor can receive the message. Neither smartphones in an elevator hall in another floor nor smartphones in another elevator hall in the same floor cannot receive the message. On receipt of the beacon message with elevator hall ID, the smartphone of the waiting user transmits the ID to the remote controller management server through $\mathrm{WiFi}$ or cellerphone networks. The remote controller management server accesses its database with the elevator hall ID and achieves the current floor number of the smartphone and a set of floor numbers the user can reach. A message with this information is sent back to the smartphone and the user interface screen is configured as shown in Figure 6. For example in this figure, the current floor of the user is 6th floor and the user can move to the 1st, 2nd, ..., 10th floors. The user can specify his/her destination floor by pushing one of the buttons on the smartphone screen. Then the destination floor is notified to the elevator controller server and the specified elevator car for the user is notified to the smartphone and displayed on the screen as shown in Figure 6.

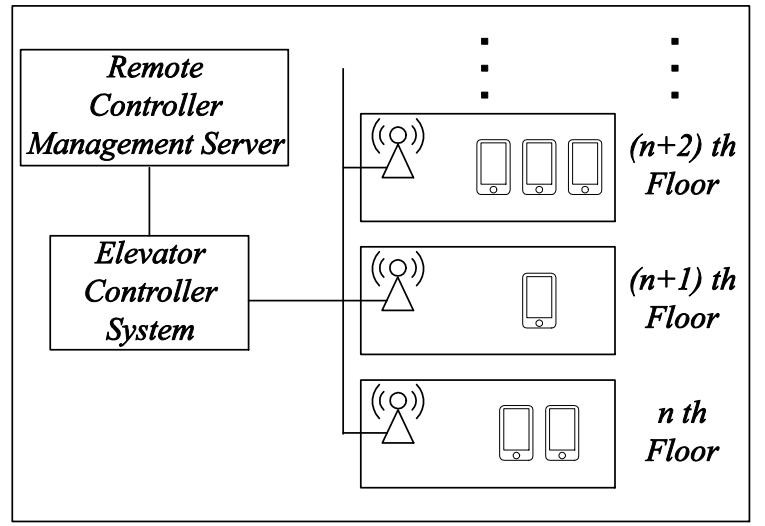

Figure 5. Proposed Elevator System.

Figure 7 shows the protocol for our proposed elevator system. In our protocol, since each user notifies the request for the elevator car to the elevator controller system, it achieves the number of users and his/her destination. In addition, the elevator controller system specifies the elevator car to each user to which he/she gets into. Hence, the elevator controller realizes more detailed control especially multiple elevator cars can be stopped at the same floor simultaneously according to the user's demand.

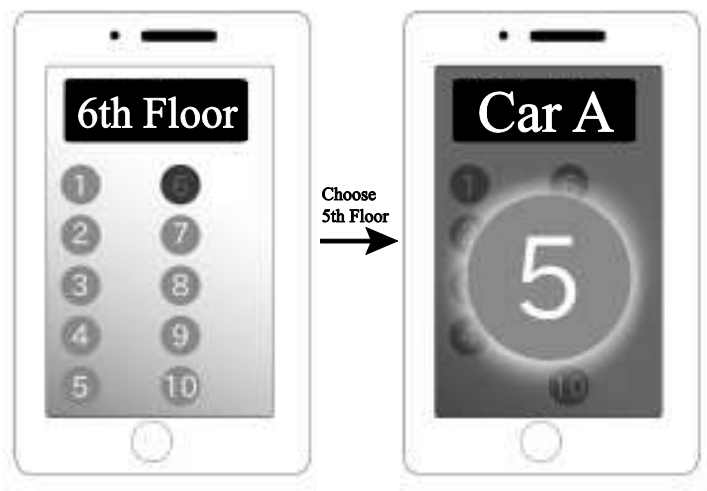

Figure 6. User Interface Provided by Remote Controller Application.

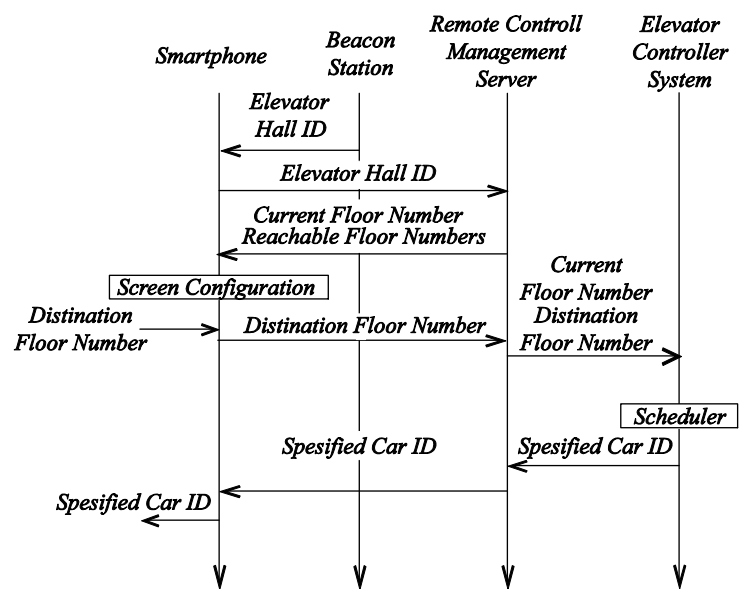

Figure 7. Protocol for Proposed Elevator System.

\section{EVALUATION}

In order to evaluate the performance of our proposed method with the user interface on user's smartphones and the remote controller management server, the smartphones and the remote controller management server are connected to an elevator simulator through the Internet. In our simulation, the conventional method and our proposed method are applied to a 10 floor building with 4 elevator cars whose capacity is 18 persons. Here, 1,500-3,200 users per hour arrives at the ground and the 1st floors according to the Poisson Process. The simulation results, i.e. average waiting time for each user, are shown in Figures $8-11$. Figures 8 and 9 show the results of the conventional method in the ground and the 1st floors respectively. Less than 2,700 users per hour arrivals can be supported without bumped passengers; however, it is difficult for the conventional method to support more than 2,700 users per hour arrivals and the waiting time of the users rapidly increased as the arrival ratio becomes high in both the ground and the 1st floors. On the other hand, figures 10 and 11 show the results of the proposed method in the ground and the 1st floors respectively. Even though the arrival ratio of the users becomes high, the waiting time of the users only 
proportionally increases with small inclination. This performance improvement is provided by the simultaneous stops at the same floor of multiple elevator cars which becomes possible by the proposed method.

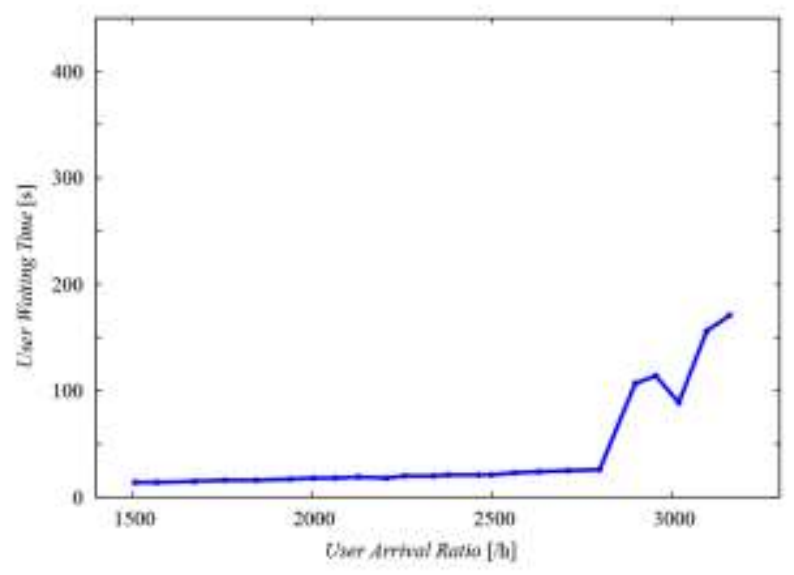

Figure 8. Simulation Result (Conventional: Ground Floor).

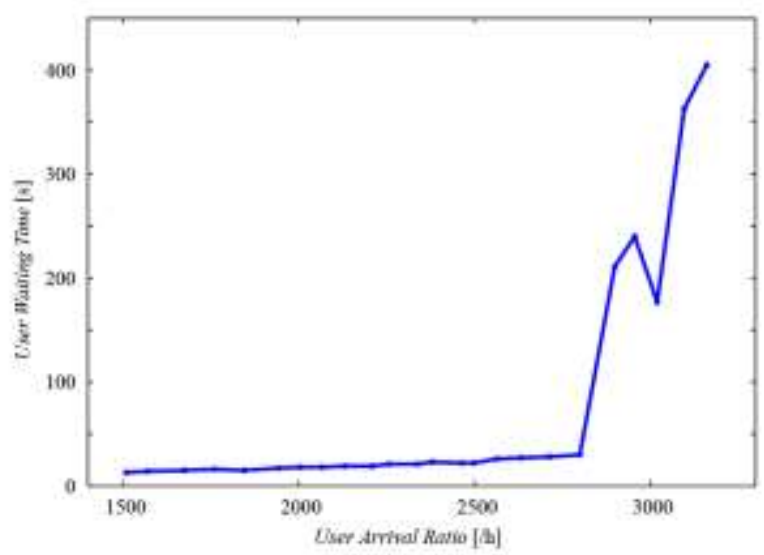

Figure 9. Simulation Result (Conventional: 1st Floor).

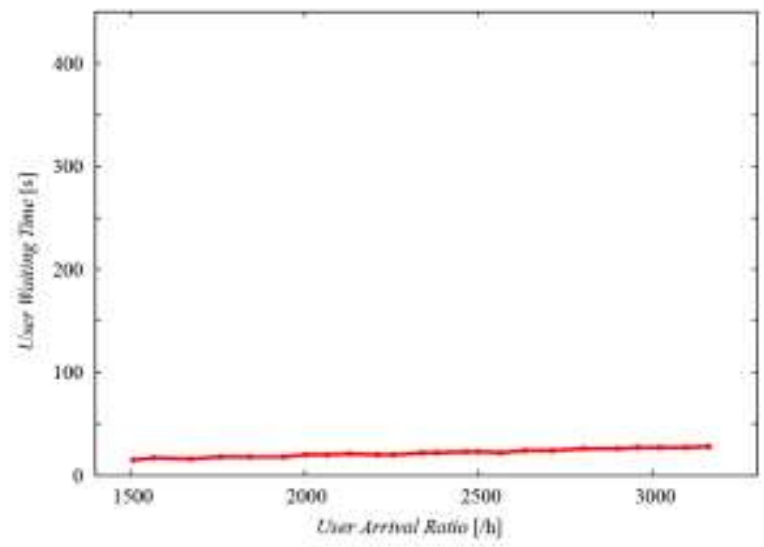

Figure 10. Simulation Result (Proposal: Ground Floor).

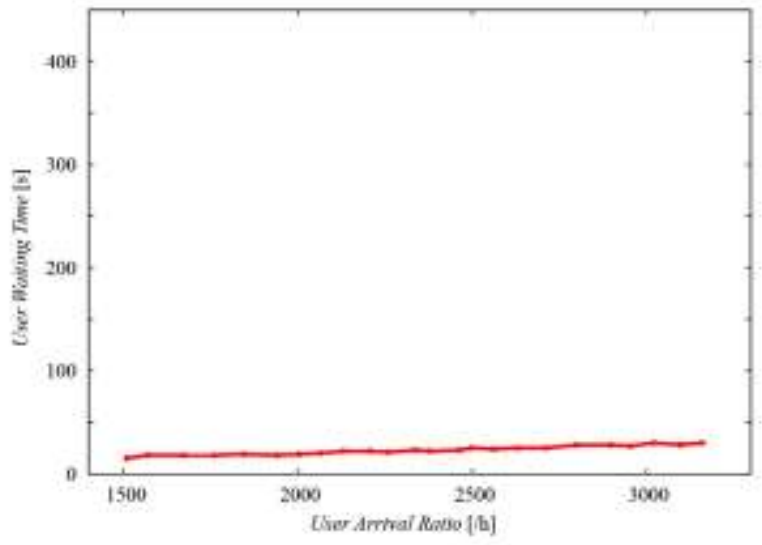

Figure 11. Simulation Result (Proposal: 1st Floor).

\section{v. CONCLUSION}

This paper proposed the novel elevator systems with smartphones of users and stationary beacon stations. By using user's smartphones as a user interface to the elevator controller system, the system achieves individual requests so that the schedule of the elevator cars is determined and modified in accordance of the detailed information of the user's requests. User's location information is achieved by using the stationary beacon stations and is notified to the elevator controller system through $\mathrm{WiFi}$. Hence, the proposed system can be implemented by using only widely available products. Simulation experiments show that our proposed method supports users with higher arrival ratio than the conventional one.

\section{References}

[1] Hikita, S., Iwata, M. and Abe, S., "Elevator Group Control with Destination Call Entry and Adaptive Control," Journal of the Institute of Electrical Engineering of Japan, vol. 124-C, no. 7, pp. 1471-1477 (2004).

[2] Hirasawa, K., Kuzunuki, S., Iwasaka, T., Kaneko, T. and Kawatake, K., "Hall Call Assignment in Elevator Supervisory Control," Journal of the Institute of Electrical Engineering of Janap, Vol. 99-C, no. 2, pp. 27-32 (1979).

[3] Kurosawa, K., Hirasawa, K., Kuzunuki, S., Yoneda, K., Sakai, Y. and Haginaka, H., "Intelligent and Supervisory Control for Elevator Group," Journal of Information Processing Society of Japan, vol. 26, no. 2, pp. 278--287 (1985).

[4] K. Elissa, "Title of paper if known," unpublished 'Guideline for Pedestrian Mobility Support Services,"

http://www.mlit.go.jp/common/000205501.pdf (2013).

About Author (s)

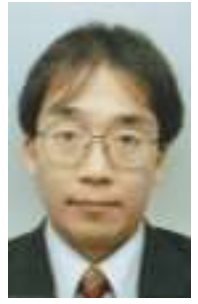

Hiroaki Higaki was born in 1967. He received his B.E. degree from Tokyo University in 1990 and D.E. degree from Tokyo Denki University in 1997, respectively. He is now a professor in Tokyo Denki University. His research interest includes mobile ad-hoc networks, wireless

sensor network, distributed systems and network protocols. He is n ACM, IEICE 